Check for updates

Cite this: RSC Adv., 2019, 9, 6143

\title{
Smartphone-based kanamycin sensing with ratiometric FRET†
}

\author{
Saurabh Umrao, ${ }^{a}$ Anusha $S,{ }^{a}$ Vasundhara Jain, $\neq^{a}$ Banani Chakraborty ${ }^{a}$ \\ and Rahul Roy (D) *abc
}

Smartphone-based fluorescence detection is a promising avenue for biosensing that can aid on-site analysis. However, quantitative detection with fluorescence in the field has been limited due to challenges with robust excitation and calibration requirements. Here, we show that ratiometric analysis with Förster resonance energy transfer (FRET) between dye pairs on DNA aptamers can enable rapid and sensitive kanamycin detection. Since our detection scheme relies on ligand binding-induced changes in the aptamer tertiary structure, it is limited only by the kinetics of ligand binding to the aptamer. Our FRET-based kanamycin binding aptamer (KBA) sensor displays two linear ranges of $0.05-5 \mathrm{nM}$ (detection limit of $0.18 \mathrm{nM}$ ) and $50-$ $900 \mathrm{nM}$ of kanamycin. The aptamer displays high specificity even in the presence of the 'natural' background from milk. By immobilizing the aptamer in the flow cell, our KBA sensor design is also suitable for repeated kanamycin detection. Finally, we show that the ratiometric FRET-based analysis can be implemented on a cheap custom-built smartphone setup. This smartphone-based FRET aptamer scheme detects kanamycin in a linear range of 50-500 nM with a limit of detection (LOD) of $28 \mathrm{nM}$.

Received 6th December 2018 Accepted 12th February 2019

DOI: $10.1039 / \mathrm{c} 8 \mathrm{ra10035g}$

rsc.li/rsc-advances analysis. Several of these methods that display high sensitivity, also rely on antibodies for analysis, which can suffer from batch-tobatch variations, high costs and limitations on scalability.

One biosensor based approach that addresses some of these limitations relies on DNA aptamers. Aptamers are single-stranded oligonucleotides, which recognize and bind to their target (ranging from small molecules to large proteins) with very high affinity and specificity. ${ }^{\mathbf{8} 9}$ Consequently, aptamers are gaining popularity in the field of biosensing. ${ }^{10}$ Since the discovery of the kanamycin binding aptamer (KBA), ${ }^{11}$ there have been several attempts to develop KBA based sensors for the detection of residual kanamycin using different optical ${ }^{12-21}$ and electrochemical ${ }^{22-33}$ schemes. Out of these, fluorescent aptasensors have several advantages due to their simpler implementation schemes and high sensitivity. Fluorescence-based aptamer detection can be classified into two major categories. The first group employs binding of a ligand to unlabelled aptamers that change fluorescence signatures (or levels) of an interacting dye. For example, organic dyes such as thiazole orange, ${ }^{34} \mathrm{~N}$-methyl mesoporphyrin, ${ }^{35}$ and thioflavin $\mathrm{T},{ }^{36}$ that selectively bind to G-quartets pocket of the KBA can be used to detect kanamycin by displacement of the dye from the aptamer leading to fluorescence changes.

However, the requirement for specific binding of the dye to the aptamer limits the universality of this aptamer approach. Alternate schemes employ fluorophore-conjugated aptamers. Such fluoro-aptasensors coupled to carbon nanotubes, ${ }^{37}$ carbon-dots, $^{38}$ nanoparticles ${ }^{\mathbf{1 9}}$ (and acting as quenchers) can detect kanamycin by the recovery of fluorescence signals upon kanamycin binding.

\footnotetext{
Department of Chemical Engineering, Indian Institute of Science, Bangalore, 560012, +91-80-2293-3118

${ }^{b}$ Molecular Biophysics Unit, Indian Institute of Science, Bangalore, 560012, India ${ }^{c}$ Center for Biosystems Science and Engineering, Indian Institute of Science, Bangalore, 560012, India

$\dagger$ Electronic supplementary information (ESI) available. See DOI: 10.1039/c8ra10035g

\$ Department of Biotechnology, Indian Institute of Technology, Roorkee, India, 247667.
} 
Table 1 DNA oligonucleotides and corresponding modifications utilized in the current study

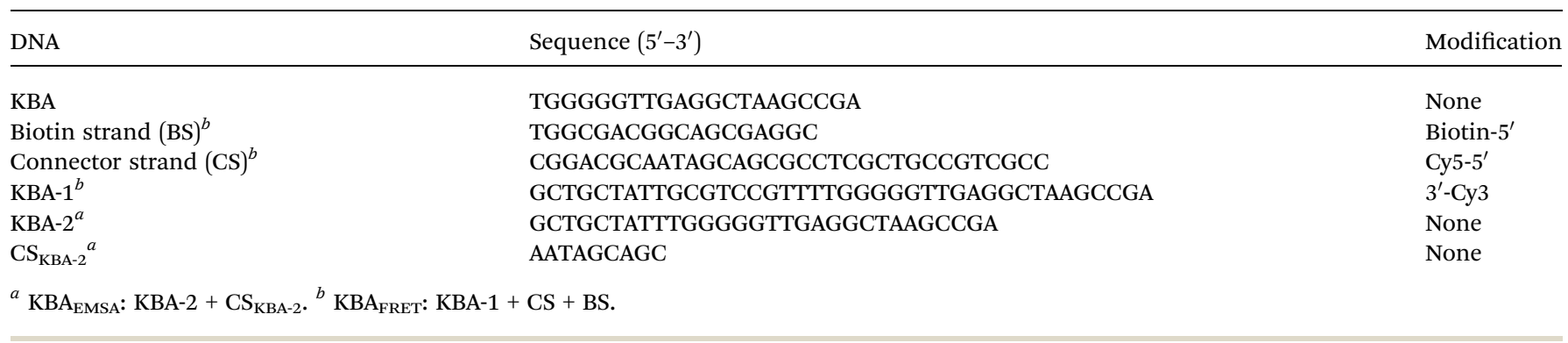

In spite of such existing fluorescence assays for detection of kanamycin using aptamers, challenges remain in their deployment in the field. Many assays still rely on fluorescence spectrophotometers or microscopes for optical readouts to ensure reliable and sensitive quantification. They also require prior fluorescence signal calibration to accurately estimate the target concentrations. To address these issues for in-the-field applications, there is a growing interest to develop robust and cost-effective optical assays and platforms that require minimal calibration.

Global availability of low cost, compact and robust complementary-metal-oxide-semiconductor (CMOS) sensors on smartphones with powerful microprocessors are an attractive option for adaptation as hand-held optical point-of-care (POC) devices. ${ }^{39}$ Smartphones have been previously used to probe thrombin activity in blood ${ }^{40}$ and quantification of mercury ions in water. ${ }^{41}$ However, fluorescence assays on smartphone devices require optimization for excitation, detection modes and calibrations. For example, intensity changes in excitation light source, inadequate focusing, optical misalignment can result in deviation of the measurements in the field. These could be avoided by using reference dyes ${ }^{42}$ but such schemes require additional light sources. Therefore, fluorescence-based biosensing using smartphones has remained limited.

One approach that can alleviate several of the challenges with implementation of fluorescence assays for smartphonebased detection is ratiometric Förster resonance energy transfer (FRET). ${ }^{43,44}$ FRET-based detection has several advantages over single color and reference dye-based detection. First, only donor dye excitation is required to collect fluorescence signal from the donor (via direct excitation) and acceptor (via energy transfer). Due to the conservation of energy, the ratio of fluorescence signal from the acceptor to the total signal from the acceptor and donor (defined here as FRET ratio) is independent of the changes in the level or wavelength of excitation. Also, variability in the concentration of the aptamer is inherently corrected for in ratiometric FRET assays. This allows repeated use of the reporter molecule in an immobilised scheme (where losses during washes or due to photobleaching will otherwise result in loss of the signal). Again, since FRET efficiency ratio is independent of orientation of the molecules, immobilized or free-floating molecules should report consistent performance across various platforms. However, the implementation of simultaneous donor-acceptor fluorescence detection for FRET determination is challenging on smartphones due to the small spectral difference between common dye pairs. In a recent report, a ratiometric FRET approach was recently reported for kanamycin detection. ${ }^{45}$ However, since the assay was dependent on DNA amplification, it relied on reagents like DNA polymerase and dNTPs and required more than 2 hours for kanamycin detection.

Here, we developed a fast and quantitative assay for kanamycin detection by integrating a well-studied kanamycin DNA aptamer $(\mathrm{KBA})^{11}$ using two-color ratiometric FRET. By placing the donor-acceptor dye pair on the aptamer, our fluorescent KBA aptamer displays a monotonic drop in FRET efficiency as a function of kanamycin concentration within seconds without any need for processing or incubation. Further, due to the simplicity of our approach, we could adapt the FRET aptasensor with a custom-made two-color fluorescence detection setup using a smartphone camera. Using our smartphone FRET device, we could detect kanamycin in a linear range of 50-500 nM.

\section{Material and methods}

\subsection{DNA aptamer design}

We have used a previously reported sequence of 21-mer kanamycin binding aptamer (KBA) ${ }^{11}$ in our study to design the kanamycin FRET sensor (Table 1). HPLC-purified, dye-labeled

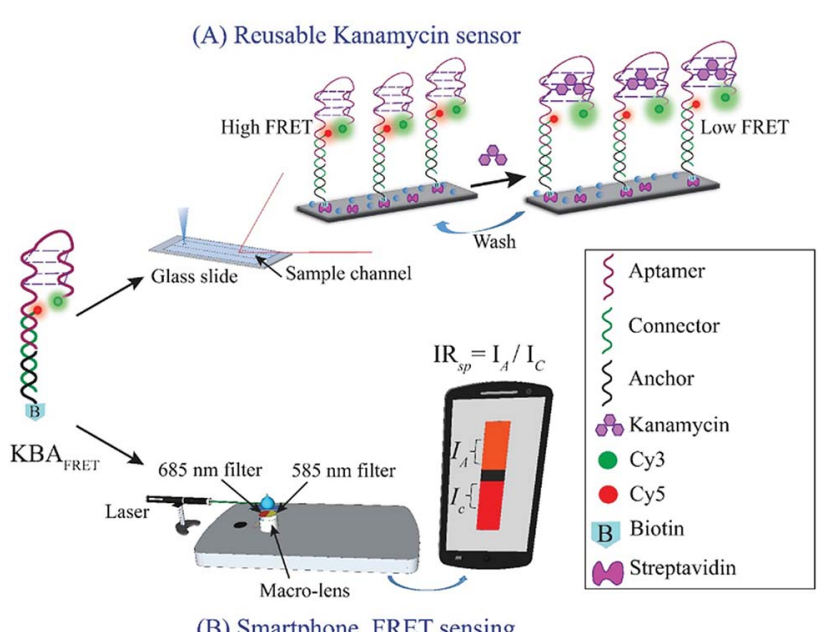

(B) Smartphone FRET sensing

Scheme 1 Schematic for FRET-based kanamycin binding aptasensor. The top half (purple strand) of the aptasensor is the kanamycin binding aptamer and the bottom double-stranded DNA region (green and black strands) is used for surface mobilization when required. (A) Surface

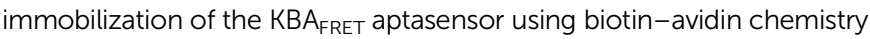
allows repeated kanamycin detection and aptasensor regeneration by washing. (B) Kanamycin detection using KBA $\mathrm{KRET}_{\text {is }}$ is demonstrated with a smartphone-based two-color fluorescence reader. 
or amino-modified (3AmMC6T) DNA were procured from Integrated DNA Technologies. Desalted unlabelled DNA strands were procured from Sigma Aldrich. The oligonucleotide sequences and their corresponding modifications are summarized in Table 1.

$N$-Hydroxysuccinimide ester Cy3 (GE Healthcare) was used to label amino-modified KBA-1 strand. Briefly, $4 \mu \mathrm{l}$ of DNA stock in water $\left(25 \mu \mathrm{g} \mathrm{\mu l}^{-1}\right)$ was mixed with $14 \mu \mathrm{l}$ of Cy3 dye $(22 \mathrm{mM}$ in dimethyl sulfoxide) and $79 \mu \mathrm{l}$ of freshly prepared $0.1 \mathrm{M}$ sodium tetraborate buffer $(\mathrm{pH}=8.5)$. The reaction mixture was incubated for $6 \mathrm{~h}$ in the dark on a rotating mixer at room temperature. Labeled DNA was recovered by ethanol precipitation. Single-stranded unlabelled aptamer, KBA was used for circular dichroism (CD) spectroscopy. KBA-2, an extended version of KBA contained 9 additional bases at the $5^{\prime}$-end to enable hybridization to a complementary connector strand, $\mathrm{CS}_{\mathrm{KBA}-2}$.
This partial duplex KBA construct (KBA $\mathrm{KMSA}_{\mathrm{EA}}$ ), as well as the single-stranded KBA-2 oligonucleotide was used for the electrophoretic mobility shift assay (EMSA). The FRET construct $\left(\mathrm{KBA}_{\mathrm{FRET}}\right)$ contained a Cy5 dye on the $5^{\prime}$-end of a connector strand (CS) apart from a Cy3 on the $3^{\prime}$-end of KBA-1 strand. These two oligonucleotides were further annealed to a biotinylated oligo (BS) to surface-immobilize the $\mathrm{KBA}_{\mathrm{FRET}}$ construct by the creation of a three-strand DNA complex.

\subsection{Non-denaturing gel electrophoretic mobility shift assay (EMSA)}

KBA-2 and $\mathrm{KBA}_{\mathrm{EMSA}}(10 \mu \mathrm{M}$ each) were incubated with $500 \mu \mathrm{M}$ kanamycin for $2 \mathrm{~h}$ at $4{ }^{\circ} \mathrm{C}$ and run on a 10\% PAGE in TBE buffer at $4{ }^{\circ} \mathrm{C}(100 \mathrm{~V}$ for $2 \mathrm{~h})$. The gels were stained with ethidium bromide and imaged on a generic UV-transilluminator gel-doc system.
(A)

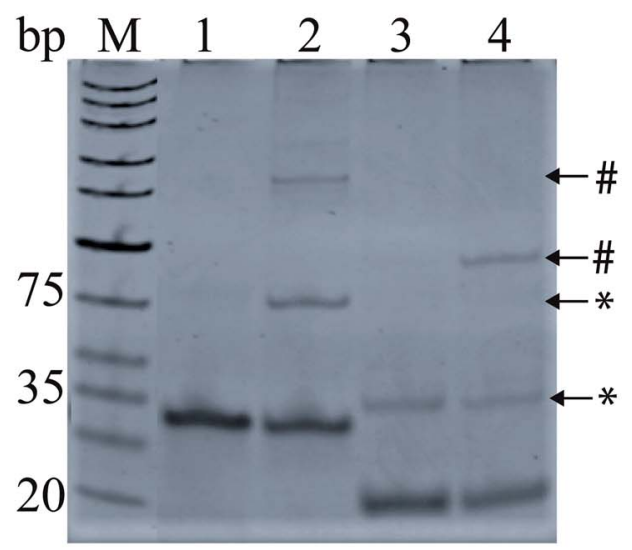

(C)

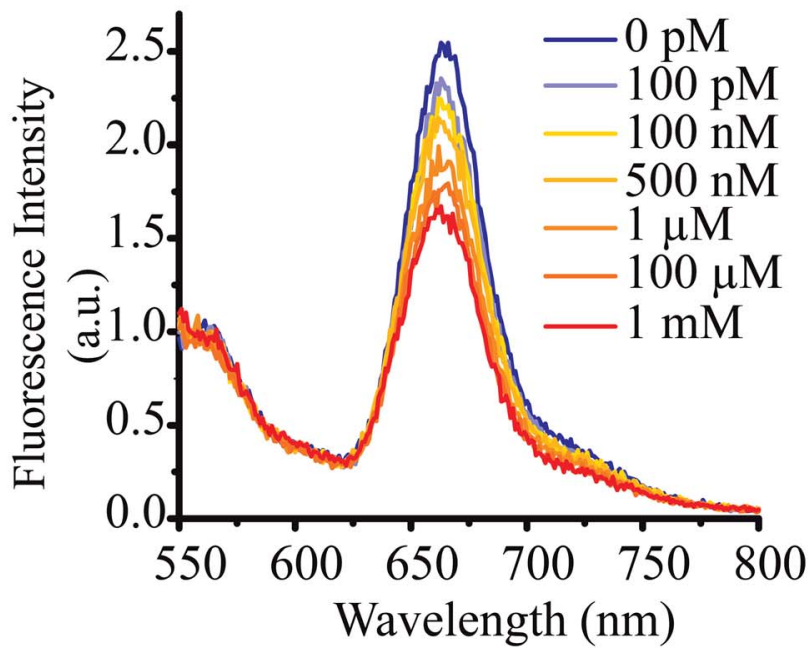

(B)

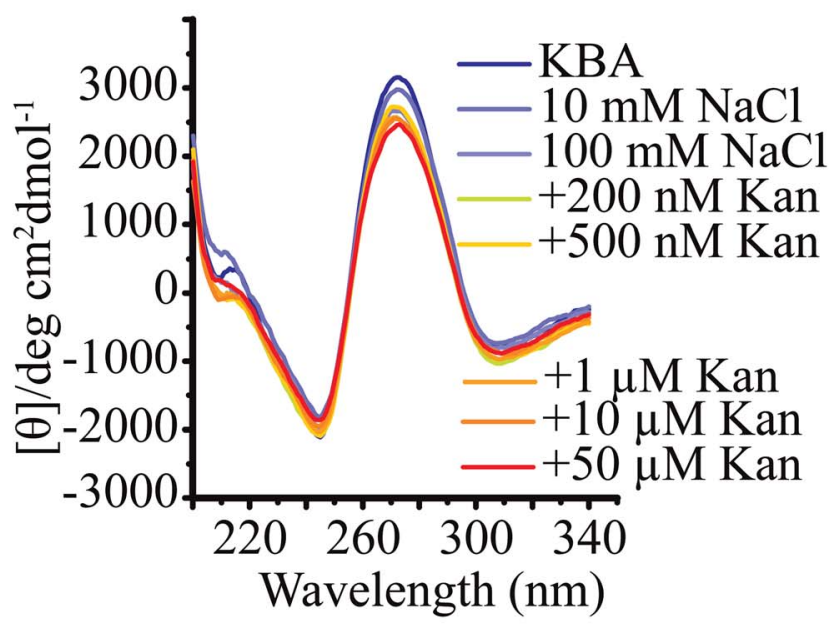

(D)

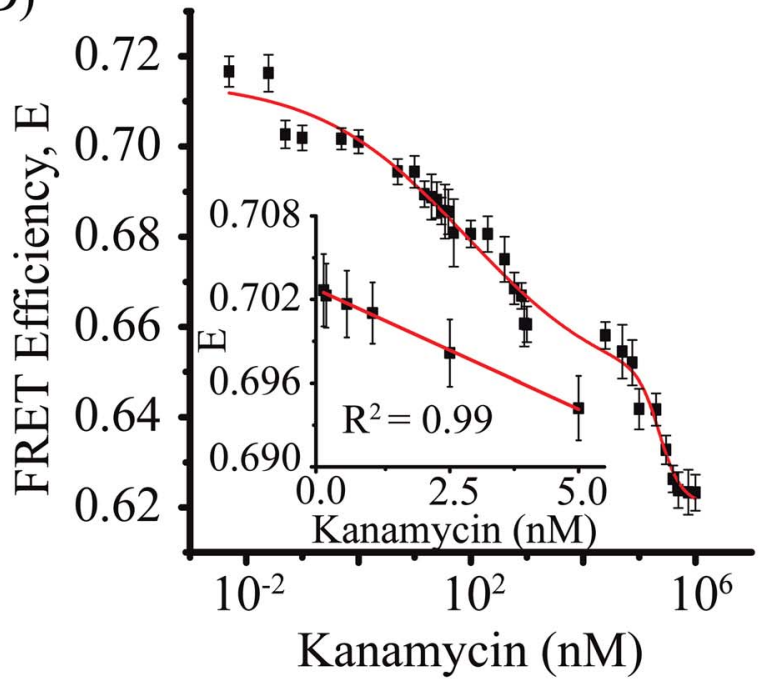

Fig. 1 KBA binding with kanamycin. (A) EMSA gel image of KBA $A_{E M S A}$ and KBA-2. Lane 1: KBA kanamycin. *Represent DNA bands corresponding to intermolecular complexes and ${ }^{*}$ represent kanamycin bound intermolecular complexes. (B) $C D$ spectra of $4 \mu \mathrm{M} \mathrm{KBA}$ with or without kanamycin (Kan). (C) Fluorescence emission spectra normalized to donor fluorescence amplitude for KBA $\mathrm{FRET}$ at different kanamycin concentrations. (D) FRET efficiency change of KBAFRET as a function of kanamycin concentrations and fit to two-site binding model

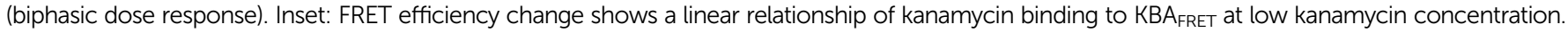




\subsection{Circular dichroism spectroscopy}

A JASCO-610 CD spectrophotometer with a $1 \mathrm{~mm}$ path length of quartz cell was used to record the circular dichroism (CD) spectra for $4 \mu \mathrm{M}$ KBA strands in $50 \mathrm{mM}$ Tris buffer $(\mathrm{pH}=7.4)$ with a range of salt and target concentrations as indicated in Fig. 1B. Reported CD spectra represent an average of six scans (340-220 nm) at room temperature.

\subsection{Fluorescence spectroscopy}

Agilent Cary Eclipse fluorescence spectrophotometer was used for the ensemble fluorescence experiments. A $530 \mathrm{~nm}$ wavelength (10 $\mathrm{nm}$ window) excitation was used for the Cy3-Cy5 FRET pair on the aptamer and emission spectra were collected for 550-800 $\mathrm{nm}$. All experiments were conducted with $2 \mathrm{nM}$ $\mathrm{KBA}_{\text {FRET }}$ at room temperature when not listed otherwise. Kanamycin (Sigma Aldrich), and other antibiotics such as streptomycin, chloramphenicol, ethambutol, ampicillin, penicillin (P)-streptomycin, tetracycline and rifamycin (Sigma Aldrich) were used at the concentrations indicated in Fig. 2B. $1 \times$ KBA buffer (50 mM Tris-HCl, $10 \mathrm{mM} \mathrm{NaCl}, \mathrm{pH} 7.4$ ) was used in all the experiments unless mentioned otherwise. For evaluating the performance of $\mathrm{KBA}_{\mathrm{FRET}}$ in presence of milk sample, we pre-treated the milk with $20 \%$ acetic acid to adjust the $\mathrm{pH}$ to 4.6, followed by incubation for $10 \mathrm{~min}$ at $45{ }^{\circ} \mathrm{C}$. The curdled milk particles were removed by centrifugation at $12000 \mathrm{rpm}$ for $20 \min ^{46}$

\subsection{The immobilized aptamer FRET assay}

Glass slides (BLUE STAR) and coverslips (No. 1, VWR International) were used for fluorescence imaging after extensive cleaning as described previously. ${ }^{47}$ A sample chamber was created by sealing the coverslip with the glass slide (with predrilled holes) using a double-sided tape as a spacer followed with epoxy resin (14270, Devcon) added to the edges. This chamber was incubated with $0.25 \mathrm{mg} \mathrm{ml}{ }^{-1}$ biotin labeled bovine serum albumin (A8549, Sigma-Aldrich) for $10 \mathrm{~min}$. After washing with T50 buffer (10 mM Tris, $50 \mathrm{mM} \mathrm{NaCl,} \mathrm{pH} 8.0$ ),
$0.2 \mathrm{mg} \mathrm{ml}^{-1}$ of streptavidin (85 878, Sigma-Aldrich) was added (10 minutes). Excess streptavidin was flushed out with T50 buffer and $2 \mathrm{nM} \mathrm{KBA}_{\mathrm{FRET}}$ was added to the chamber to immobilize them to the surface (for $10 \mathrm{~min}$ ) followed by washing the chamber with $400 \mu \mathrm{l}$ of $1 \times$ KBA buffer. We used $50 \mathrm{nM}$ protocatechuate-3,4-dioxygenase (P8279, Sigma-Aldrich), $2.5 \mathrm{mM}$ protocatechuic acid (03930590, Sigma-Aldrich) and 1 mM 6-hydroxy-2,5,7,8-tetramethylchromane-2-carboxylic acid (238 813, Sigma-Aldrich) as an oxygen scavenging system to reduce the photo-bleaching of $\mathrm{KBA}_{\mathrm{FRET}}$ construct. Images of immobilized aptamer were acquired using a custom-built twocolor imaging setup ${ }^{48}$ on an Olympus IX81 inverted microscope that employs a solid-state laser (532 nm, Sapphire SF 532$100 \mathrm{~mW}$, Coherent) to excite Cy3 fluorophore (10-50 mW at the laser head). The sample was excited with a highly-inclined angle of illumination to reduce the out-of-focus noise. ${ }^{49}$ The fluorescence signal is collected by an oil immersion objective $(100 \times$, 1.4 numerical aperture (NA) oil objective, UplanSApo100XO, Olympus) using a long pass filter (E550LP, Chroma Tech.), multi-notch filters (NF01-405/488/532/635, Semrock). A vertical slit in the image plane was used after the long pass filter to restrict the imaging area such that the final image occupies half of the electron multiplying charge-coupled device (EMCCD) camera chip. An image of this region (from the slit) is relayed by a pair of $10 \mathrm{~cm}$ focal length lenses to the camera plane. A dichroic mirror (FF555-Di02-25 $\times 36$, Semrock) was used to split the donor and acceptor fluorescence emissions after the first lens and the focused images for both were focused by the second lens and collected side-by-side on the same chargecoupled device imaging sensor (iXon Ultra 897, DU-897U-CS0BV, Andor Technology).

\subsection{Smartphone-based two-color fluorescence detection}

The setup integrates three components (i) a compact battery powered class III green laser (Amazon, Inc) to produce an excitation (532 $\pm 10 \mathrm{~nm}, 100 \mathrm{~mW}$ ) (ii) a polyacrylic sample slide cradle $\left(40 \times 30 \times 35 \mathrm{~mm}^{3}\right)$ designed using CorelDRAW software and cut with a laser engraving machine (VLS 3.60, ULS Inc.) and
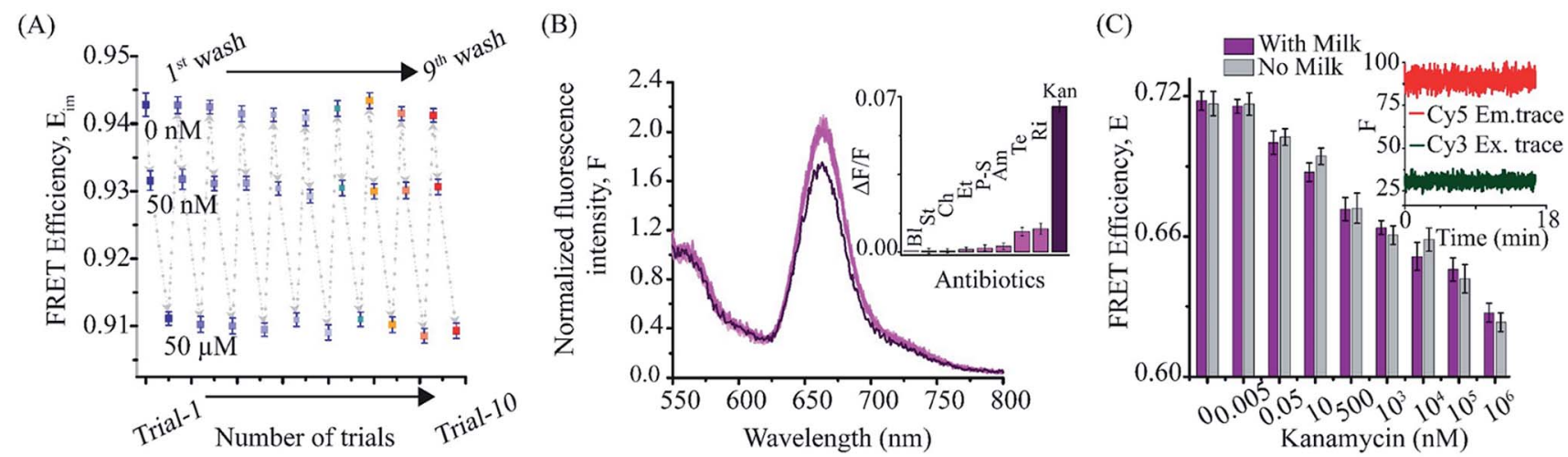

Fig. 2 FRET aptasensor reusability and selectivity (A) FRET efficiency change for KBAFRET with kanamycin is plotted for ten trials (shown in different colors). (B) Normalized fluorescence spectra of KBAFRET in presence of various antibiotics. Inset: $\Delta F / F$ for respective antibiotics (each at $100 \mu M$; kanamycin at $100 \mathrm{nM}$ ), Bl (blank), St (streptomycin), Ch (chloramphenicol), Et (ethambutol), Am (ampicillin), P-S (penicillin-streptomycin), Te

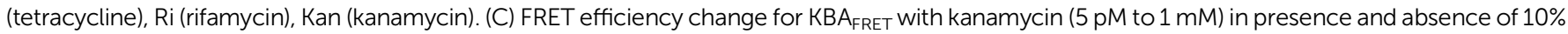

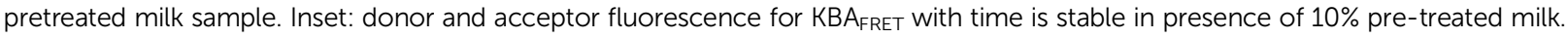


(iii) a $200 \times$ external macro lens attached on a Redmi Note 4 camera-phone (Xiaomi) with appropriate filters. The laser diode was mounted $\sim 60 \mathrm{~mm}$ away from the sample chamber and the height of the laser was adjusted to illuminate the sample at close to zero-degree incidence angle. To overcome the limitations of the SNR and excitation light leakage, the sample was illuminated from the side and fluorescence was collected orthogonal to the excitation. The cradle protects the user from laser exposure and improves the signal to noise ratio (SNR) by restricting stray light. To collect the two-color emission signal from the sample slide, the two halves of the image collected by the macro lens were spectrally selected using two acrylic color filters (COMAR Optics). The top half of the image was blocked with a 685 long pass filter (685AY50) to acquire the acceptor only emission and the bottom half carried a 585 long pass filter (585AY50) to collect the donor plus acceptor emission. Leakage from the area near the junction of the filters was eliminated by placing an opaque tape over the region. This imaging geometry generates a spectrally split fluorescent image of the $\mathrm{KBA}_{\mathrm{FRET}}$ solution on the smartphone camera (as depicted in Scheme 1 and ESI Fig. S1†).

\subsection{Data analysis}

To compare the fluorescence changes under different conditions, measured intensity spectra were normalized to the fluorescence intensity at $570 \mathrm{~nm}$, the maximum emission wavelength of Cy3 and plotted. FRET (efficiency) ratio $(E)$ was calculated by taking the ratio of acceptor intensity $\left(I_{\mathrm{A}}\right)$ to the sum of acceptor and donor intensity $\left(I_{\mathrm{A}}+I_{\mathrm{D}}\right)$. Hill equation fit $(n=1)$ was used to recover the dissociation constants $\left(K_{\mathrm{d}}\right)$ for intermolecular KBA complexes and all errors reported for dissociation constants represent fitting errors. Fractional fluorescence changes $(\Delta F / F)$ was calculated from $\left(F-F_{0}\right) / F_{0}$, where $F$ and $F_{0}$ correspond to the fluorescence intensity of the aptamer with and without the presence of kanamycin, respectively. The theoretical LOD for kanamycin was calculated using expression $3.3 \times(\sigma / s)$ with a $95 \%$ confidence interval as per IUPAC guidelines. Here, $\sigma$ and $s$ refer to the standard deviation of $y$-intercepts of the regression line and slope of the linear fit respectively.

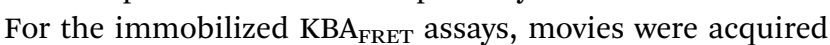
at $100 \mathrm{~ms}$ per frame integration time on the EMCCD using the Andor Solis acquisition software. Movies were analyzed with the ImageJ software to generate average fluorescence intensities (from the first 20 frames for the selected regions of interest) of Cy3 and Cy5 fluorophores after background correction. Apparent FRET efficiency $\left(E_{\text {im }}\right)$ for the immobilized aptamers was calculated as an acceptor to total intensity ratio. Note that this apparent FRET efficiency was not corrected for the transmission efficiency and spectral bandwidth of the filters or the spectral dependence of the quantum efficiency of the CCD camera and hence the $E_{\text {im }}$ does not match the FRET efficiency acquired on the fluoro-spectrophotometer.

For the smartphone-based FRET assay, the image of region of interest that was illumination by the laser excitation was analyzed. The two spectrally distinct regions spanning a total of 510 pixels in the length and 86 pixels in a non-spectral direction (width) was processed to estimate the fluorescence intensity ratio. A line selection was made across the two spectrally split images and intensity values from region $\mathrm{C}$ and $\mathrm{A}$ (Fig. 3A) was used to calculate the average pixel intensities of acceptor emission $\left(I_{685}\right)$ and total fluorescence emission $\left(I_{585}\right)$, respectively. Next, we separated the corresponding R-G-B channel intensities for the images from region $\mathrm{C}$ and $\mathrm{A}$. A smartphone fluorescence intensity ratio $\left(\mathrm{IR}_{\mathrm{sp}}\right)$ was calculated using the expression, $\mathrm{IR}_{\mathrm{sp}}=I_{685, \mathrm{R}} / I_{585, \mathrm{G}}$, where $I_{685, \mathrm{R}}$ was the red channel intensity from the region C and $I_{585, \mathrm{G}}$ was the green channel intensity from the region A. Background correction values were estimated with the same setup but without any sample in the sample chamber and applied for each region separately before $\mathrm{IR}_{\mathrm{sp}}$ calculation. The analysis was done using ImageJ. ${ }^{50}$

\section{Results and discussion}

To develop a rapid-response FRET-based aptasensor for detection of kanamycin, we evaluated whether a ligand-induced change in aptamer conformation can be employed to report on ligand binding. Such structural changes can range from folding of the aptamer from an unfolded form or changes in the secondary structure (that involves rearrangement of base pairing) and/or transformations in the tertiary structure. The latter is most pertinent when ligand binding is contingent upon pre-formation of a binding pocket in the folded aptamer. ${ }^{34}$ Measuring changes in the tertiary structure for such aptamers provide a fast assay format since it is largely dependent on the binding rate constant of the ligand. However, it is not clear if such tertiary structural changes in the aptamer can produce detectable signals (e.g. in FRET efficiency change) to be viable as a biosensor approach.

\subsection{Detecting kanamycin using intramolecular KBA G- quadruplex formation}

To develop a FRET assay that reports on aptamer conformation, we first investigated the kanamycin aptamer (KBA) structure.
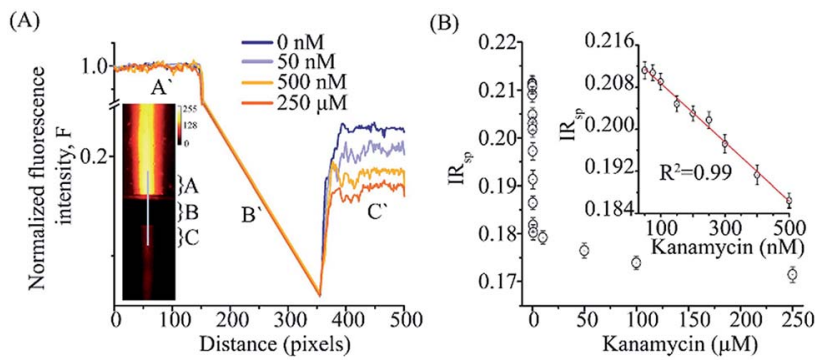

Fig. 3 KBA aptasensor on a smartphone platform (A) KBA FRET sample imaged (inset) using two-color smartphone imager shows $585 \mathrm{~nm}$ long-pass $\left(/_{585}\right)$ emission at the top (including the segment A) and $685 \mathrm{~nm}$ long-pass $\left(I_{685}\right)$ emission at bottom (including the segment $C$ ). The dark region $B$ is due to the blocking tape. Intensity profile (white line) was used to extract the fluorescence values in A, B and C. Fluorescence intensity of subsections were normalized to intensity of section $A$ and represented as $A^{\prime}, B^{\prime}$ and $C^{\prime}$, respectively. Plot shows the normalized KBA $A_{F R E T}$ fluorescence emission with different concentrations of kanamycin. (B) Fluorescence intensity ratio $\left(I R_{s p}\right)$ change for $\mathrm{KBA}_{\mathrm{FRET}}$ with titration of kanamycin $(25 \mathrm{nM}$ to $250 \mu \mathrm{M})$ in plotted. $\mathrm{IR}_{\mathrm{SP}}$ shows a linear relationship with kanamycin binding to KBA $\mathrm{FRET}_{\text {. }}$. 
KBA has been reported to form a four-stranded intermolecular G-quadruplex structure at room temperature. ${ }^{34}$ However, the intermolecular G-quadruplex (GQ) formation has been reported at high concentrations (in the range of 1-10 $\mu \mathrm{M}$ ) of aptamer DNA, which can be a limitation in several ways. Apart from the higher costs associated with using such concentration of aptamers, changes in measured FRET signal can arise from binding of kanamycin to the GQ reporter and/or changes in the inter-molecular GQ equilibrium in the presence of kanamycin and hence results might be difficult to interpret.

To simplify our data interpretation, we decided to first characterize intermolecular GQ formation and then employ conditions that favor the intramolecular GQ form in our $\mathrm{KBA}_{\mathrm{FRET}}$ assays. We first examined intermolecular GQ formation using EMSA. When we performed EMSA for the KBA-2 (containing only the aptamer oligo sequence), we observed the formation of an intermolecular G-quadruplex structure as reported previously ${ }^{34}$ (Fig. 1A) by the appearance of a slow migrating gel band (highlighted with * in lane 3 ). In the presence of kanamycin, an additional lower mobility band is observed in lane 4 (highlighted with \#). The large reduction in the mobility of this aptamer band suggests further oligomerization of the DNA aptamer upon addition of kanamycin, possibly by stabilization of the higher order structures. Similarly, the $\mathrm{KBA}_{\mathrm{EMSA}}$ construct (KBA containing a partial duplex and mimics the FRET aptasensor) also demonstrated the presence of two distinct intermolecular complexes upon addition of kanamycin, suggesting that the incorporation of a partial duplex at the end of the aptamer did not perturb the aptamer intermolecular G-quadruplex. It is worth noting that this happened in spite of a reduction in $\mathrm{KBA}_{\mathrm{EMSA}}$ intermolecular Gquadruplex form in absence of kanamycin.

We further confirmed the conformation of the kanamycin aptamer by circular dichroism (CD) spectroscopy (Fig. 1B). KBA in the low salt condition ( $10 \mathrm{mM} \mathrm{NaCl})$ displayed a positive peak at $270 \mathrm{~nm}$ and a negative peak at $245 \mathrm{~nm}$, a characteristic CD spectrum displayed by intermolecular parallel G-quadruplexes. ${ }^{51,52}$ Therefore, we observed that KBA forms stable Gquadruplex structures at room temperature even in low salt solutions consistent with previous reports. ${ }^{34}$ Addition of kanamycin $(0.2-50 \mu \mathrm{M})$, however, did not cause any significant change in the amplitude or peak wavelength of CD spectra. This suggested that the binding of kanamycin results in only minor structural changes in the KBA G-quadruplex that is imperceptible by CD spectroscopy. It is worth noting that methods like EMSA and CD spectroscopy require aptamers in the micro-molar range and observed the formation of intermolecular complexes at these concentrations is consistent with earlier reports. ${ }^{34,53}$

Since, intermolecular complex formation can be slow, we examined if structural changes in KBA upon kanamycin binding can be reported by intramolecular FRET. We designed a FRET-

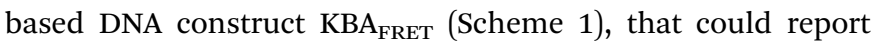
both changes upon ligand binding (through tertiary folding of the aptamer) as well as intermolecular GQ formation (due to intermolecular FRET).

We first examined if the $\mathrm{KBA}_{\mathrm{FRET}}$ could report the formation of intermolecular G-quadruplex structures. KBA $\mathrm{KRET}_{\text {fisplayed }}$ a high FRET efficiency, $E(\sim 0.69$, calculated as the ratio of acceptor to the sum of donor and acceptor signals) even at concentrations as low as $2 \mathrm{nM}$ (ESI Fig. S2A $\dagger$ ) where little or no intermolecular complexes are expected. Such a high FRET state is indicative of the formation of at least a partially folded form of the aptamer driven by intramolecular hydrogen bonding. ${ }^{54}$ However, increasing the $\mathrm{KBA}_{\mathrm{FRET}}$ concentration led to a further increase in FRET until it saturated at $E=0.86$ (at $50 \mathrm{nM}$ of $\mathrm{KBA}_{\text {FRET }}$ ) (Fig. S2A $\dagger$ inset).

Next, we examined if the intermolecular complexes formed at high concentrations are further stabilized upon kanamycin binding. First, we titrated increasing amounts of unlabelled KBA-2 DNA aptamer with respect to KBA FRET $(15 \mathrm{nM})$. As anticipated KBA-2 exchanged with the labeled DNA aptamer molecules in the intermolecular G-quadruplexes, this was confirmed by a decrease in the FRET values from $E=0.84$ to 0.79 (ESI Fig. S2B $\dagger$ ). When the same titration experiment is carried out in presence of $5 \mu \mathrm{M}$ of kanamycin, less than half of this decrease in FRET could only be recovered. Therefore, we conclude that kanamycin binding stabilized the KBA $\mathrm{FRET}_{\text {DNA }}$ in the intermolecular complex. Cations such as $\mathrm{Na}^{+}$and $\mathrm{K}^{+}$are also known to stabilize G-quadruplex structures. ${ }^{55}$ In order to optimize the salt conditions, we studied the effects of these salts upon kanamycin aptamer folding, we measured the FRET signal from $\mathrm{KBA}_{\mathrm{FRET}}$ as a function of salt concentration (ESI Fig. S2C $\dagger$ ). Interestingly, the NaCl-induced aptamer stabilization lead to a biphasic reduction in the FRET signal $(E=0.73$ at $10 \mathrm{mM} \mathrm{NaCl}$ to $E=0.67$ at $1 \mathrm{M} \mathrm{NaCl}$ ) suggesting that the stable form of the folded aptamer results in the dye-pair at the ends of the aptamer to move away from each other compared to the initial folded state. $\mathrm{KCl}$ induced a slightly lower level of FRET $(E=0.66$ at $1 \mathrm{M}$ $\mathrm{KCl})$ consistent with reports that $\mathrm{K}^{+}$stabilizes G-quadruplex structures better than $\mathrm{Na}^{+} \cdot{ }^{52,56}$ However, the location of inflection points at similar concentrations for both salts and high concentration of salts required to reach saturation suggests that non-specific electrostatic screening is the dominant aptamer stabilizing mechanism.

This demonstrated that a careful characterization of the aptamer structure in different solution and conditions is required to correctly interpret the FRET signals observed. Based on our studies above, we performed all subsequent kanamycin binding measurements using $2 \mathrm{nM}$ KBA ${ }_{\text {FRET }}$ construct in $10 \mathrm{mM} \mathrm{NaCl}$ (optimized conditions). By carefully selecting the FRET sensor concentration, we are able to attribute the FRET change to kanamycin induced changes in KBA structural changes.

When kanamycin ( $5 \mathrm{pM}$ to $1 \mathrm{mM}$ ) was added to KBA FRET under optimized solution conditions, a monotonic drop in the FRET values with increasing kanamycin concentration was observed (Fig. 1C and D). The drop in the FRET is consistent with the previous experiments where the addition of salts is also supposed to stabilize the KBA structure (compare ESI Fig. S2C $\dagger$ ). The drop in FRET efficiency (instead of an increase) is due to the unique placement of our dyes on the aptamer. The FRET efficiency values could be best fit with a two-binding site model as a function of kanamycin concentration. The lower dissociation constant, $K_{\mathrm{d} \text {, квA,1 }}=89.05 \pm 26.23 \mathrm{nM}$ 
matched well with the previously reported $K_{\mathrm{d}}$ for the same KBA suggesting that the dye attached to the aptamer did not perturb kanamycin binding. ${ }^{11}$ The second dissociation constant, $K_{\mathrm{d}, \mathrm{KBA}, 2}=229.66 \pm 7.14 \mu \mathrm{M}$ suggested the possible binding of second kanamycin to the aptamer. Such binding of two kanamycin molecules to kanamycin aptamer combined with the FRET method results in a large dynamic (spanning from the picomolar to micromolar) range for our KBA FRET biosensor. The linear range of kanamycin detection falls within two kanamycin concentration ranges of $0.05-5 \mathrm{nM}$ (Fig. 1D inset) and 50-900 nM (ESI Fig. S3†) with the lowest detection limit of $0.18 \mathrm{nM}$. This shows that FRET reporters based on organic dye-conjugated DNA aptamers can be sensitive biosensors even if the aptamer is pre-folded.

In parallel, we tested kanamycin quantification using the standard microbiological assay test. E. coli (K-12) bacterial strain was subjected to different concentrations of kanamycin and their growth rate was monitored (ESI Fig. S4A $\dagger$ ). Bacterial concentration $\left(\mathrm{OD}_{600}\right)$ fit to Gompertz model ${ }^{57}$ gave an minimum inhibitory concentration median $\left(\mathrm{MIC}_{50}\right)$ value of $4.7 \mu \mathrm{g} \mathrm{ml}^{-1}$ (ESI Fig. S4B $\dagger$ ) comparable to previous reports. ${ }^{\mathbf{5 8 , 5 9}}$ Since the DNA duplex and G-quadruplex folding are sensitive to temperature, we also measured the FRET efficiency of the $\mathrm{KBA}_{\text {FRET }}$ at different temperatures (30-90 $\left.{ }^{\circ} \mathrm{C}\right)$. Indeed, we

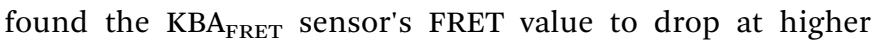
temperatures (ESI Fig. S6†). Interestingly, the aptamer continues to detect kanamycin even at $60{ }^{\circ} \mathrm{C}$ (ESI Fig. S6† inset). In this work, we performed all kanamycin calibration and measurements at $25{ }^{\circ} \mathrm{C}$ to avoid complications in data interpretation at different temperatures. Our FRET-based method reports an inexpensive detection scheme for kanamycin with comparable or better performance in terms of sensitivity and dynamic range to those reported standard detection methods (ESI Table S1†). Moreover, our 'one-pot' FRET biosensor requires fewer steps and can report kanamycin concentrations with rapid response times (complete in $\sim 3 \mathrm{~s}$ at $100 \mathrm{pM}$ kanamycin, ESI Fig. S5† inset).

\subsection{Reusable KBA biosensor with surface immobilization}

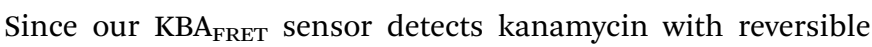
structural changes, we further employed a surfaceimmobilization scheme to evaluate its reusability. We immo-

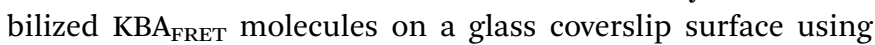
biotin-streptavidin binding. A monotonic drop in the FRET efficiency was recorded during kanamycin binding to KBA $\mathrm{FRET}$ construct as observed in solution FRET measurements (Fig. 2A). We used the $1 \times$ KBA buffer to wash the bound kanamycin and non-specifically bound $\mathrm{KBA}_{\mathrm{FRET}}$ molecules from the aptamer

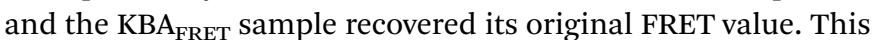
recovery of same FRET value upon washing further confirmed that we were observing intramolecular FRET change due to kanamycin binding to the aptamer. Repeated cycles of kanamycin titration with $\mathrm{KBA}_{\mathrm{FRET}}$ displayed insignificant differences in the FRET efficiency reported for each concentration tested. This exemplifies the use of ratiometric FRET as a robust measurement compared to other methods where measured signal is proportional to aptamer concentration and hence subject to change upon loss of sensor molecules during the assays. Note that the high apparent FRET $\left(E_{\text {im }}\right)$ values observed on the microscope arise from the differences in camera and fixed wavelength filter based detection and quantum efficiencies for the donor and acceptor dyes compared to the spectrophotometer. Nevertheless, once calibrated for any two-color ratiometric optical setup, the apparent FRET values are not expected to change.

\subsection{Selectivity of KBA FRET sensors}

We further tested the selectivity of $\mathrm{KBA}_{\mathrm{FRET}}$ assay against seven antibiotics (including, streptomycin, chloramphenicol, ethambutol, penicillin-streptomycin, ampicillin, rifamycin and tetracycline, each at $100 \mu \mathrm{M}$ concentration). FRET changes were insignificant for these antibiotics, compared to kanamycin (100 nM) (Fig. 2B). Next, the KBA aptasensor was tested for the detection of kanamycin in milk samples. To test the integrity of KBA $_{\text {FRET }}$ molecules in milk samples, we added $10 \%$ of pretreated milk to the KBA FRET. We observed a stable fluorescence signal for both dyes (constant FRET) over $15 \mathrm{~min}$, suggesting minimal degradation of the aptasensor in presence of milk constituents (Fig. 2C, inset). To test kanamycin detection in milk samples, pre-treated milk samples were spiked with different concentrations of kanamycin. KBA FRET assay was tested with these samples and FRET efficiency was compared to the standard (without milk) kanamycin solutions (Fig. 2C). The signals were comparable and we observed only a minor loss in detection sensitivity from the FRET signatures in the milkspiked samples compared aptamers in the buffer.

\subsection{Kanamycin detection using a smartphone-based fluorescence reader}

Ratiometric FRET detection also enables deployment of our aptasensor in assays where robust illumination or optical detection might be a challenge. We adapted the KBA FRET aptasensor assay to a smartphone-based two-color fluorescence detection platform to make it more accessible for resourcelimited and in-the-field environments. We simplified the spectrophotometer based aptamer FRET measurement by replacing the (i) xenon flash lamp (as commonly used in commercial fluorospectrometers) with a battery powered green laser diode pointer as the excitation source, (ii) multiple sets of optics with a single external collection lens $(\sim 200 \times$ magnification with smartphone camera) and (iii) the commercial monochromator with two acrylic long-pass filters that allowed us to limit emission detection to desired spectral windows. Our setup allowed us to scale down the capital cost of the aptamer FRET detection to a low-cost device ( $\sim 23$ USD plus the cost of the smartphone)

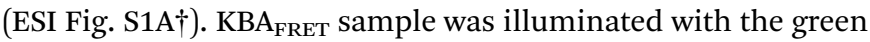
laser diode and fluorescence images from the sample chamber were collected using the smartphone camera. Side-by-side split detection of two spectral windows (due to the two filters placed on the camera sensor) allowed ratiometric FRET estimation (Fig. 3A and ESI Fig. S1B $\dagger$ ). Inset of Fig. 3A shows the picture captured on the smartphone that is divided into two spectral 
regions and intensity ratio $\left(\mathrm{IR}_{\mathrm{sp}}\right)$ was calculated as described in data analysis section. We could robustly detect FRET loss in $\mathrm{KBA}_{\text {FRET }}$ molecules upon kanamycin binding by calculating the $\mathrm{IR}_{\mathrm{sp}}$ as a concentration-dependent signal drop in the acceptor spectral window (Fig. 3B). Recorded intensity ratio, $\mathrm{IR}_{\mathrm{sp}}$ from the smartphone displayed a linear range of kanamycin detection over 50-500 nM with a LOD of $28.06 \mathrm{nM}$ (Fig. 3B, inset).

\section{Conclusions}

In summary, our study demonstrates a sensitive, selective and easy to implement FRET aptamer assay for rapid 'one-pot' detection of kanamycin. We show that the pre-folded state of the aptamers displays further conformational changes upon target binding that enables quantification of ligand concentration. The aptamer can be reused with surface immobilization without loss in sensitivity due to its ratiometric nature. Further, we have demonstrated a portable, cost-effective and sensitive smartphone based ratiometric FRET platform. While the calculated FRET ratios were different across various platforms (namely, spectrophotometer, fluorescence microscope and smartphone imager), the FRET aptasensor remarkably provided consistent results due to the ratiometric nature of the assay. We propose that the developed FRET-based ratiometric device holds promise for different POC applications including use with existing aptamers for other target detection assays.

\section{Conflicts of interest}

There are no conflicts to declare.

\section{Acknowledgements}

We would like to acknowledge Mr Satyaghosh Maurya for his assistance in setting up microbiological assay for kanamycin. This research was supported by Indian Institute of Science at Bangalore, Department of Science and Technology, Government of India grant to RR (SR/S3/CE/079/2012) and Ramalingaswami Fellowship from Department of Biotechnology, Government of India to BC (BT/RFL/RE-ENTRY/13/2013). SU also acknowledges the support of Newton-Bhabha Fellowship (BT/IN/UK/DBT-BC/2015-16).

\section{Notes and references}

$1 \mathrm{WHO}$, Implementation of the global action plan on antimicrobial resistance, WHO GAP AMR Newsletter No. 32, 2017.

2 A. Forge and J. Schacht, Audiol. Neuro-Otol., 2000, 5, 3-22.

3 L. P. Rybak and C. A. Whitworth, Drug Discovery Today, 2005, 10, 1313-1321.

4 EMEA, European Agency for the Evaluation of Medical Products (EMEA) London, Regulation No. EMEA/MRL/886/ 03-FINAL, 2003.

5 Y. X. Zhou, W. J. Yang, L. Y. Zhang and Z. Y. Wang, J. Liq. Chromatogr. Relat. Technol., 2007, 30, 1603-1615.
6 M. Frasconi, R. Tel-Vered, M. Riskin and I. Willner, Anal. Chem., 2010, 82, 2512-2519.

7 E. E. M. G. Loomans, J. Van Wiltenburg, M. Koets and A. Van Amerongen, J. Agric. Food Chem., 2003, 51, 587-593.

8 C. Tuerk and L. Gold, Science, 1990, 249, 505-510.

9 A. D. Ellington and J. W. Szostak, Nature, 1990, 346, 818-822.

10 W. Z. Zhou, P. J. J. Huang, J. S. Ding and J. Liu, Analyst, 2014, 139, 2627-2640.

11 K. M. Song, M. Cho, H. Jo, K. Min, S. H. Jeon, T. Kim, M. S. Han, J. K. Ku and C. Ban, Anal. Biochem., 2011, 415, 175-181.

12 R. Y. Robati, A. Arab, M. Ramezani, F. A. Langroodi, K. Abnous and S. M. Taghdisi, Biosens. Bioelectron., 2016, 82, 162-172.

13 M. Ramezani, N. M. Danesh, P. Lavaee, K. Abnous and S. M. Taghdisi, Sens. Actuators, B, 2016, 222, 1-7.

14 Y. Y. Xu, T. Han, X. Q. Li, L. H. Sun, Y. J. Zhang and Y. S. Zhang, Anal. Chim. Acta, 2015, 891, 298-303.

15 C. B. Liu, C. X. Lu, Z. G. Tang, X. Chen, G. H. Wang and F. X. Sun, Microchim. Acta, 2015, 182, 2567-2575.

16 L. S. Khabbaz, M. Hassanzadeh-Khayyat, P. Zaree, M. Ramezani, K. Abnous and S. M. Taghdisi, Anal. Methods, 2015, 7, 8611-8616.

17 J. Chen, Z. H. Li, J. Ge, R. Yang, L. Zhang, L. B. Qu, H. Q. Wang and L. Zhang, Talanta, 2015, 139, 226-232.

18 S. C. Niu, Z. Z. Lv, J. C. Liu, W. H. Bai, S. M. Yang and A. L. Chen, PLoS One, 2014, 9, e109263.

19 H. Li, D. E. Sun, Y. J. Liu and Z. H. Liu, Biosens. Bioelectron., 2014, 55, 149-156.

20 K. H. Leung, H. Z. He, D. S. H. Chan, W. C. Fu, C. H. Leung and D. L. Ma, Sens. Actuators, B, 2013, 177, 487-492.

21 Z. P. Liu, C. S. Tian, L. H. Lu and X. G. Su, RSC Adv., 2016, 6, 10205-10214.

22 F. L. Li, X. Y. Wang, X. Sun and Y. M. Guo, $R S C A d v .$, 2017, 7, 38981-38988.

23 N. D. Zhou, J. B. Luo, J. Zhang, Y. D. You and Y. P. Tian, Anal. Methods, 2015, 7, 1991-1996.

24 M. Zhao, Y. Zhuo, Y. Q. Chai and R. Yuan, Biomaterials, 2015, 52, 476-483.

25 Y. M. Xin, Z. Z. Li and Z. H. Zhang, Chem. Commun., 2015, 51, 15498-15501.

26 X. L. Qin, W. J. Guo, H. J. Yu, J. Zhao and M. S. Pei, Anal. Methods, 2015, 7, 5419-5427.

27 R. Liu, Z. H. Yang, Q. Guo, J. C. Zhao, J. Ma, Q. Kang, Y. F. Tang, Y. Xue, X. H. Lou and M. He, Electrochim. Acta, 2015, 182, 516-523.

28 W. Xu, Y. Wang, S. Liu, J. H. Yu, H. Z. Wang and J. D. Huang, New J. Chem., 2014, 38, 4931-4937.

29 X. Sun, F. L. Li, G. H. Shen, J. D. Huang and X. Y. Wang, Analyst, 2014, 139, 299-308.

30 R. Z. Li, Y. Liu, L. Cheng, C. Z. Yang and J. D. Zhang, Anal. Chem., 2014, 86, 9372-9375.

31 J. Dapra, L. H. Lauridsen, A. T. Nielsen and N. Rozlosnik, Biosens. Bioelectron., 2013, 43, 315-320.

32 Y. Zhu, P. Chandra, K. M. Song, C. Ban and Y. B. Shim, Biosens. Bioelectron., 2012, 36, 29-34. 
33 A. A. Rowe, E. A. Miller and K. W. Plaxco, Anal. Chem., 2010, 82, 7090-7095.

34 Y. P. Xing, C. Liu, X. H. Zhou and H. C. Shi, Sci. Rep., 2015, 5, 8125.

35 H. L. Yang, Q. H. Wu, D. X. Su, Y. Wang, L. Li and X. P. Zhang, Anal. Sci., 2017, 33, 133-135.

36 L. Ma, N. N. Sun, C. H. Tu, Q. Zhang and A. P. Diao, RSC Adv., 2017, 7, 38512-38518.

37 Q. G. Liao, B. H. Wei and L. G. Luo, Microchim. Acta, 2017, 184, 627-632.

38 Y. S. Wang, T. C. Ma, S. Y. Ma, Y. J. Liu, Y. P. Tian, R. N. Wang, Y. B. Jiang, D. J. Hou and J. L. Wang, Microchim. Acta, 2017, 184, 203-210.

39 M. Zarei, TrAC, Trends Anal. Chem., 2017, 91, 26-41.

40 E. Petryayeva and W. R. Algar, Analyst, 2015, 140, 4037-4045.

41 Q. S. Wei, R. Nagi, K. Sadeghi, S. Feng, E. Yan, S. J. Ki, R. Caire, D. Tseng and A. Ozcan, ACS Nano, 2014, 8, 11211129.

42 A. N. Kapanidis and S. Weiss, J. Chem. Phys., 2002, 117, 10953-10964.

43 H. J. Yu, Y. F. Tan and B. T. Cunningham, Anal. Chem., 2014, 86, 8805-8813.

44 P. R. Selvin, Biochemical Spectroscopy, 1995, vol. 246, pp. 300334.

45 J. K. Deng, Y. Q. Liu, X. D. Lin, Y. L. Lyu, P. C. Qian and S. Wang, Sens. Actuators, B, 2018, 273, 1495-1500.

46 N. D. Zhou, J. Zhang and Y. P. Tian, Anal. Methods, 2014, 6, 1569-1574.
47 S. D. Chandradoss, A. C. Haagsma, Y. K. Lee, J. H. Hwang, J. M. Nam and C. Joo, J. Vis. Exp., 2014, e50549, DOI: 10.3791/50549.

48 R. Roy, S. Hohng and T. Ha, Nat. Methods, 2008, 5, 507-516. 49 M. Tokunaga, N. Imamoto and K. Sakata-Sogawa, Nat. Methods, 2008, 5, 159-161.

50 C. A. Schneider, W. S. Rasband and K. W. Eliceiri, Nat. Methods, 2012, 9, 671-675.

51 J. Kypr, I. Kejnovska, D. Renciuk and M. Vorlickova, Nucleic Acids Res., 2009, 37, 1713-1725.

52 N. V. Hud, F. W. Smith, F. A. L. Anet and J. Feigon, Biochemistry, 1996, 35, 15383-15390.

53 S. Umrao, V. Jain, Anusha, B. Chakraborty and R. Roy, Sens. Actuators, B, 2018, 267, 294-301.

54 M. C. Murphy, I. Rasnik, W. Cheng, T. M. Lohman and T. J. Ha, Biophys. J., 2004, 86, 2530-2537.

55 I. Russo Krauss, A. Merlino, A. Randazzo, E. Novellino, L. Mazzarella and F. Sica, Nucleic Acids Res., 2012, 40, 8119-8128.

56 A. N. Lane, J. B. Chaires, R. D. Gray and J. O. Trent, Nucleic Acids Res., 2008, 36, 5482-5515.

57 R. J. W. Lambert and J. Pearson, J. Appl. Microbiol., 2000, 88, 784-790.

58 A. M. Pereira, A. C. Abreu and M. Simões, Journal of Microbiology Research, 2012, 2, 84-88.

59 G. E. Burrows, R. J. Morton and W. H. Fales, J. Vet. Diagn. Invest., 1993, 5, 541-547. 